\title{
Controversy: HLA Antibodies Responsible for Fetal/Neonatal Alloimmune Thrombocytopenia - an Update
}

\author{
Simon Panzer \\ Clinic for Blood Group Serology, Medical University of Vienna, Austria
}

\section{Key Words}

FNAITP · Platelet antibodies · HLA antibodies

\section{Summary}

This review focuses on reports and data dealing with an association between an alloimmunization against HLA class I antigens and fetal/neonatal alloimmune thrombocytopenia (FNAITP) that have been published since 2000. Current serological requirements for a presumed diagnosis of FNAITP in newborns are presented to understand the hesitation towards a diagnosis of HLA antibody-associated FNAITP. Three already published case reports are extensively discussed, and data from 3 series of presumed FNAITP are presented. Furthermore, the data on light chain restriction of such HLA antibodies are discussed in view of a possible clonality of these antibodies in presumed FNAITP.

\section{Introduction}

This review focuses on reports on fetal/neonatal alloimmune thrombocytopenia (FNAITP) - presumably due to alloimmunization to HLA antigens - published during the last 6 years. Platelets express HLA class I antigens, and HLA antibodies are known to adversely affect the success of platelet transfusions. Likewise, it is conceivable that HLA antigens can serve

\author{
Schlüsselwörter \\ FNAITP · Thrombozytenantikörper · HLA-Antikörper
}

\section{Zusammenfassung}

Diese Übersichtsarbeit soll dem Leser die Entwicklungen seit dem Jahre 2000 hinsichtlich einer Beziehung einer Immunisierung im HLA-System und der möglichen Entwicklung einer fötalen/neonatalen Alloimmunthrombozytopenie (FNAITP) näher bringen. Zunächst sind die Bedingungen, die für eine serologischen Diagnostik einer FNAITP zu erfüllen sind, angeführt. Daraus soll verständlich werden, weshalb die Annahme einer durch HLAAntikörper vermittelten FNAITP schwierig ist und in den seltensten Fällen überzeugend dargestellt werden kann. Drei publizierte Berichte von Einzelfällen werden diesbezüglich kritisch beleuchtet und die Inzidenz von HLAAntikörpern in 3 Studien von Patienten, die zur Abklärung einer FNAITP untersucht wurden, präsentiert. Darüber hinaus wird eine Studie vorgestellt, in der die Leichtketten solcher Antikörper untersucht wurden, um einer möglichen pathophysiologischen Rolle von Klonalitäten näher zu kommen. as targets leading to HLA antibody-induced FNAITP. A very comprehensive review on the subject has been published by Taaning in 2000 [1]. After carefully discussing the pros and cons, the author concluded that evidence that HLA antibodies may induce FNAITP is rather weak. Since this publication only three new papers have been dealing with neonatal thrombocytopenia which might be due to maternal alloantibodies with HLA class I specificity [2-4].

\begin{tabular}{ll}
\hline KARGER & @ 2006 S. Karger GmbH, Freiburg \\
Fax +49 7614520714 & Accessible online at: \\
$\begin{array}{l}\text { E-mail Information@Karger.de } \\
\text { www.karger.com }\end{array}$ & www.karger.com/tmh
\end{tabular}


This review will briefly summarize diagnostic requirements for the assumed diagnosis of 'classical' platelet-specific, antibodyinduced FNAITP and shall illustrate why FNAITP remains always an 'assumed' diagnosis, even if incompatibilities with regard to the established specific human platelet antigens (HPA) between the mother and child can be detected. Furthermore, it shall outline why most platelet immunologists hesitate towards a diagnosis of HLA class I-induced FNAITP even if these are the only detectable antibodies. Some aspects which have not been covered in the past and which became apparent due to newer technologies or recent overall knowledge in prenatal immunology will be discussed.

\section{Pathophysiology}

FNAITP is due to transplacental transfer of maternal IgG alloantibodies which react with an alloantigen on the fetal platelets that is inherited from the father and in the mother is lacking. NAITP occurs in about 1of 1,000 to 2,000 unselected pregnancies $[5,6]$. The most common involved alloantigen is HPA-1a, but other platelet-specific alloantigens may also serve as targets. Moreover, maternal HPA-1 alloimmunization is associated with the most severe outcome. Alloimmunization against other platelet-specific antigens are significantly less frequent. This may be due to antibodies which are not detected with current methods, be it due to difficulties like those with the known antigen system HPA-3 to specify the target $[7,8]$, be it that the target is unknown. Whatever the reason is, platelet-specific alloantibodies are detectable in only about $25-30 \%$ of sera from mothers delivered from a thrombocytopenic offspring with suspected FNAITP [9]. This rather low diagnostic achievement marks either the high number of other than alloimmune-induced mechanisms that may lead to fetal/neonatal thrombocytopenia or a high rate of FNAITP cases which are not characterized/detectable with current methods. On the other hand, facing thrombocytopenia in an offspring and the detection of platelet antigen incompatibility between mother and her baby, does not implicitly mean that these two findings, namely thrombocytopenia on the one hand and serological incompatibility between mother and offspring on the other, are associated. This conclusion is based on findings that an offspring may not be thrombocytopenic even though there is serological incompatibility with the mother. Such cases are usually only observed in screening programs because the baby will not show clinical evidence for thrombocytopenia [6]. Thus, not all fetomaternal incompatibilities (maternal antibodies bind to the platelets of the newborn) result in FNAITP, and even if all laboratory data are compatible with FNAITP it still remains a diagnosis of 'assumption'. In conclusion, FNAITP is a clinical diagnosis, strongly substantiated by specific laboratory data but not ruled out if these are negative.

\section{Prerequisites for the Assumed Diagnosis of FNAITP}

FNAITP first of all is a diagnosis of exclusion. Only after all other reasons that may contribute to thrombocytopenia in the newborn are ruled out, an immune-mediated thrombocytopenia can be assumed. Of course, rare cases may have both nonimmune and immune thrombocytopenia. The latter may be either due maternal autoimmune disorder (e.g. autoimmune thrombocytopenia) or alloimmunization. Specific serological results are needed to substantiate the diagnosis of FNAITP. An assumption of FNAITP has therefore to based on:

- exclusion of any nonimmune pathology possibly associated with thrombocytopenia in the offspring,

- established mismatch between maternal antigens and those in the baby,

- detection of a maternal antibody that reacts with the offspring's platelets allo-specificity (for the latter, paternal platelets may be used),

- successful elution of maternal antibodies from the child's platelets (or absorption/elution experiments using paternal platelets) increasing the evidence of serological incompatibility.

The failure to identify specific serological incompatibilities does not exclude a diagnosis of FNAITP, however. A variety of reasons are discussed in the next paragraph.

\section{Serological Pitfalls}

International workshops revealed some aspects that need consideration in antibody determinations [10-12]. First, titration studies of HPA-1a antibodies show that even these antibodies are detectable at different titers among various laboratories. Second, some antibodies are detected in one laboratory, but not in another one. Furthermore and possibly most important, particularly HPA-3 antibodies may be detected by one method and at the same time not by another one [8]. These variations demand that more than one method and investigations in more than only one laboratory are applied to rule out platelet-specific antibodies. The use of fresh as well as stored platelets may further increase the sensitivity. Last but not least, the target platelet specificity is not known in cases of suspected FNAITP. Therefore, the search with even the most sophisticated method may fail if the wrong targets are evaluated. This became clear when HPA-15 became known as a possible target in FNAITP. In retrospective evaluations antibodies were detected in some of so far unsolved cases of suspected FNAITP [13-15]. Fetal platelets may absorb the maternal antibody and therefore the maternal serum may appear antibody negative. Therefore, if serology from an initial sample is negative, it is highly recommended to obtain the maternal serum again about 4 weeks to 3 months after delivery. Maternal autoimmune thrombocytopenia can lead to thrombocytopenia in the child, even if the mother has a normal platelet count. Thus, a maternal platelet 
Table 1. Light chain phenotypes of maternal HLA antibodies detectable by the MAIPA assay in 17 cases of suspected FNAIT

\begin{tabular}{lllll}
\hline Platelets $\left(\times 10^{9} / 1\right) /$ & Hemorrhage & Platelet antibodies & LCT specificity ${ }^{\S}$ & Light chain phenotype \\
\hline n.t. & petechial & autoantibody & B35 & kappa/lambda \\
32 & petechial & HPA-1a & multispecific & kappa/lambda \\
50 & petechial & negative & multispecific & kappa/lambda \\
30 & petechial & HPA-5b & A2,B5 & kappa/lambda \\
n.t. & intracranial, hematoma & HPA-1a, HPA-15b & negative & kappa/lambda \\
n.t. & none & HPA-5b & negative & kappa/lambda \\
20 & petechial & negative & negative & kappa/lambda \\
n.t. & petechial & negative & negative & kappa/lambda \\
44 & petechial & negative & multispecific & lambda \\
12 & petechial, suffusions & negative & A2, A28 & lambda \\
20 & petechial & negative & A2, A28 & lambda \\
25 & petechial & HPA-1a & A2 & lambda \\
10 & petechial, hematoma & HPA-1a & B13 & lambda \\
45 & petechial & HPA-5b & B13 & lambda \\
n.t. & hematoma & negative & A9 & lambda \\
17 & petechial & HPA-1a & negative & lambda \\
n.t. & petechial & HPA-5b & negative & kappa \\
\hline
\end{tabular}

n.t. $=$ Not tested

${ }^{\S}$ All sera contained by MAIPA-detectable HLA antibodies. count within the normal range does not exclude that her autoantibodies affect the offspring's platelets. Furthermore, it has to be kept in mind that only about $50 \%$ of patients with established autoimmune thrombocytopenia have detectable autoantibodies. A test of the maternal platelets for the presence of antibody coating is therefore more reliable than just the platelet count to exclude maternal autoimmune thrombocytopenia. Only if all the above mentioned prerequisites and pitfalls have been thoroughly considered, the absence of plateletspecific incompatibility may be assumed. Only in these cases a focus on HLA class I incompatibilities is justified.

Keeping these premises in mind, this review will focus on newer data with regard to HLA class I antibodies involvement in NAITP.

\section{Reports on HLA Class I Incompatibilities and FNAITP since 2006}

Three reports have focused on HLA class I incompatibilities and FNAITP since the last review by Taaning [1] and are discussed in order of their publication.

Saito et al. [2] provided serological analysis from three cases with assumed FNAITP in association with HLA antibodies. One offspring with low weight had severe respiratory distress and low Apgar scores. Genotypes of HPA-1 to HPA-8 were determined. Serological testing by mixed passive hemagglutination assay (MPHA) included HPA-1 to HPA-6 antigens and $\mathrm{Nak}^{\mathrm{a}}$, the null allele of GPIV (Nak can be a target in Japanese individuals). Flow cytometry investigations were applied to screen for platelet/leukocyte-reactive antibodies. Maternal sera were also absorbed to paternal platelets, and these ab- sorbed sera and platelet eluates were tested. Cross-matches were carried out with paternal cells. A HPA mismatch between the mother and her baby was only seen in the first case (HPA-4). Platelet eluates from all cases contained the respective HLA antibodies, but these antibodies were not detectable by all applied HLA-specific methods. Eluates did not contain HPA antibodies. All investigations were carried out in the same laboratory using more than one detection system. Only sera obtained within 3 days after delivery were tested. Maternal autoimmune disease was only ruled out by platelet counts, and neither the maternal nor the offsprings' platelets were tested for antibody coating. The authors provide further data from other 27 cases with assumed HLA antibody-associated NAIT. Only high-titer antibodies were associated with thrombocytopenia ( $>1$ in 16). This observation is in contrast to their case 3 who had an antibody titer of 1 in 2 but severe, treatment-requiring thrombocytopenia $\left(27 \times 10^{9} / 1\right)$. All babies from cases with HLA-A or HLA-B antibodies had also leukocytopenia. HLA-C antibodies were associated with rather mild thrombocytopenias $(n=5)$.

Moncharmont et al. [3] reported a case of presumed HLA class I antibody-induced FNAITP. The diagnosis was based on the exclusion of infection. The amniotic fluid contained meconium, however, which indicates stress to the fetus. A decrease of leukocytes on day 6 was interpreted as being due to HLA antibodies which reacted not only with platelets but also with leukocytes. HPA-1, HPA-3, and HPA-5 were determined and cross-matches included glycoproteins (GP) Ib/IX, Ia/IIa, IIb/ IIIa and $\beta_{2}$ microglobulin $\left(\beta_{2} \mathrm{~m}\right)$. Absorption/elution experiments were not performed. Maternal sera were tested on day 3 , 12 , and 38 after delivery in the same laboratory, applying a screening test (Capture P; Immucor, Norcross, CA, USA) and a 
GP-specific capture assay (monoclonal antibody-specific immobilization of platelet antigens; MAIPA). Autoimmune disease of the mother was ruled out by her normal platelet count; her platelets were not tested, but the baby's platelets were not coated as determined by the screening test. According to the above mentioned criteria for a diagnosis of assumed FNAITP, infection is not ruled out satisfactorily; only a limited number of GP has been included in the cross-match investigations, and genotyping included only few HPA. Maternal autoimmune disease which may have affected the newborns' platelets was not ruled out completely. It is not clear why the baby's platelets should not be coated with IgG, be it of HLA or any other specificity.

Thude et al. [4] reported an assumed NAIT (forth child) caused by HLA B27 antibodies. Thrombocytopenia was mild, and recovery was spontaneous. There was no evidence for any causes associated with thrombocytopenia in the newborn. Platelet autoimmune disease of the mother was ruled out by her normal platelet count and a negative test for antibody coating. Genotype for HPA-1 to HPA-6 and HPA-15 were determined, and only the latter was mismatched between mother and the newborn. The presence of platelet-specific antibodies was ruled out by MAIPA using GPIb/IX, GPIIb, GPIIIa, GPIa, GPIIa, and CD109 as targets of the father's platelets. Absorption/elution experiments were not performed. All investigations were only done with sera obtained shortly after delivery in only one laboratory using a single method for the detection of HPA antibodies.

In summary, the assumed FNAITP-implicating HLA class I alloimmunization are based on inefficiently ruled out HPA antibodies and, in some cases, other reasons of thrombocytopenia in the newborn. Maternal autoimmune disease in association with thrombocytopenia in the newborn was only efficiently ruled out in the papers of Moncharmont et al. [3] and Thude et al. [4]. HPA-15 was specifically addressed in the publication by Thude et al. [4], and is mentioned in the acknowledgment of the report by Moncharmont et al. [3]. The reports are merely descriptive without investigations to explain mechanisms where HLA class I antibodies induce FNAITP.

\section{New Series on FNAITP}

Leukoagglutinins (HLA antibodies) develop in $20-30 \%$ of pregnancies in the absence of previous transfusion or significant fetomaternal blood transfer. Three large series on human platelet antigen-specific alloantibodies implicated in cases of FNAITP have been published since 2000 , which also provide data on HLA antibodies $[9,14,15]$. The first report shows that platelet-specific antibodies (including GPIV and CD36) were detected in $31 \%$ of 3,743 suspected cases referred for the evaluation of NAITP [9]. These investigation included HPA-1 to HPA-6 allotypes, but not HPA-15. HLA class I or ABO alone were found in $37 \%$. Since this is a summary of results obtained from 1990 to 2002 the investigations are not reported in
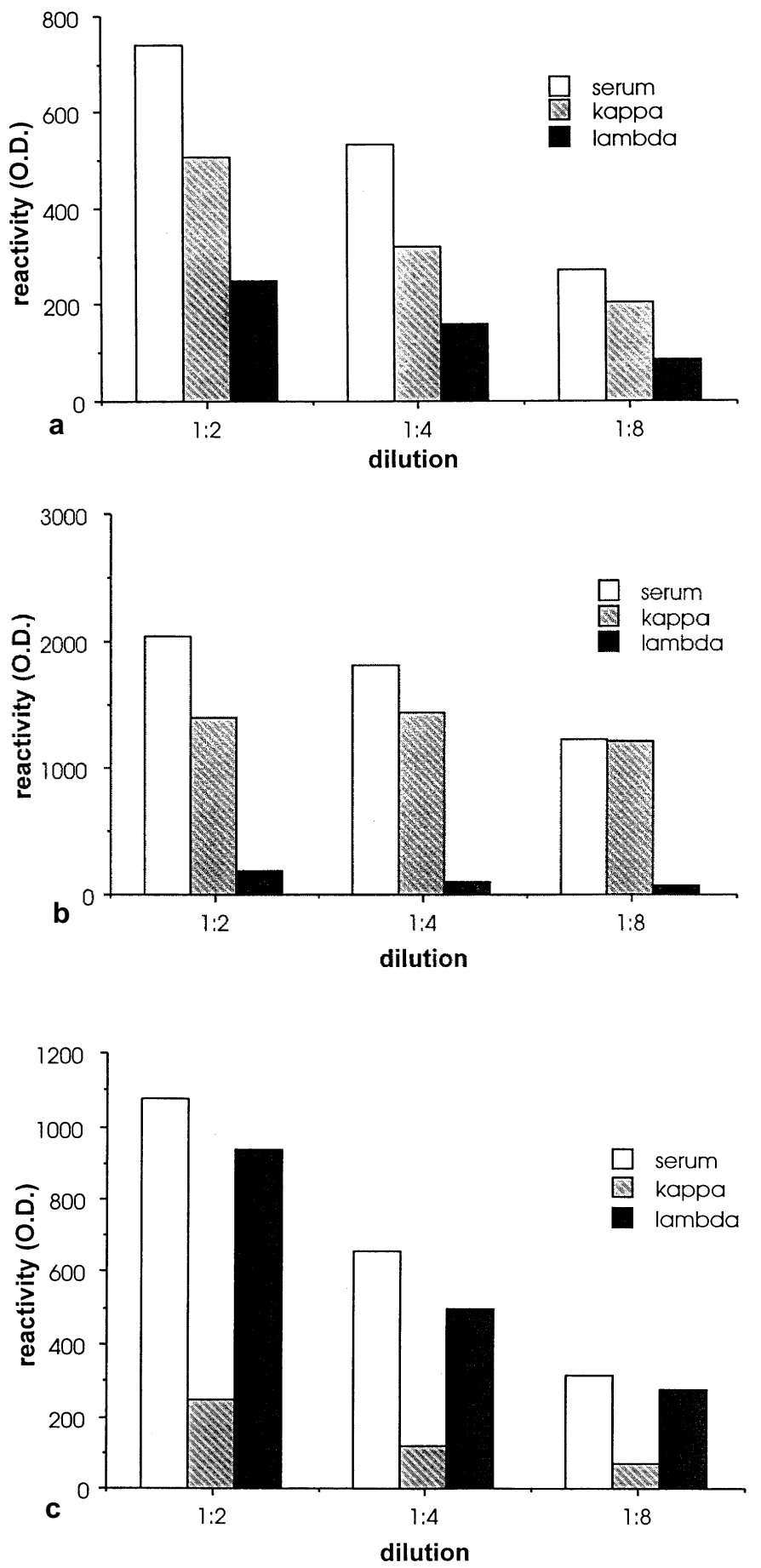

Fig. 1. Light chain-restricted reactivities of total sera, and sera after immunodepletion for either kappa or lambda antibodies were tested by MAIPA using the monoclonal antibody B1G6 (anti- $\beta_{2} \mathrm{~m}$; Immunotech, Marseille, France). A lambda phenotype was assumed if kappa/lambda was less or equal to 1.3, and a kappa phenotype if kappa/lambda was greater than 2.6. a Mixed kappa/lambda reactivities, $\mathbf{b}$ antibodies of the kappa phenotype, $\mathbf{c}$ antibodies of the lambda phenotype.

detail, and certainly not all samples were studied in full detail as desirable. The newer studies by Etel et al. [14] and Mandelbaum et al. [15] emphasized on HPA-15 alloantibodies. Their reports on HLA alloimmunization ( 26 and $41 \%$ of solitary 
HLA class I antibodies, respectively) are a byproduct. These numbers are still in the range of what is anticipated in unselected cohorts of women after delivery. Especially cases with prematurity and with small for gestational age are thrombocytopenic, and their mothers more frequently have HLA antibodies $[1,16]$. In these latter cases the underlying disease may dispose for the formation of these antibodies. Such cases with thrombocytopenia in the newborn are often referred to specialized laboratories to exclude FNAITP, without further return information. These cases are certainly contained in the cited series. The study by Mandelbaum et al. [15] comprised cases which fulfilled all serological requirements, except for the need of confirmation by a second laboratory. The high number of solitary HLA antibodies and the common co-immunization against HPA and HLA antigens prompted the investigation for clonality of HLA class I antibodies [17].

\section{Clonal Restriction of HLA Class I Antibodies}

Previous studies have focused in IgG subtypes and titers [1]. Clonal expansion can be considered to be specific for a driving antigen, namely the HLA molecule on the platelet, pointing towards a specific response to epitopes on the platelet surface. A feasible method to screen for clonal restriction is to determine light chain phenotypes of antibodies. We have tested maternal sera from 17 cases with suspected FNAITP who all met the requirements to confirm/exclude FNAITP by MAIPA using anti-human light chain-specific antibodies [17]. All sera were also tested after immunodepletion of either kappa or lambda antibodies. For this exploratory study mono- and multispecific HLA antibodies were used with or without concomitant HPA-1, or HPA-5, or HPA-15 antibodies. The crossmatch with paternal platelets was positive in all cases due to
HLA antibodies. Five, three and three sera contained lymphocytotoxic antibodies of single, double and multiple specificities, respectively. In 6 cases, HLA antibodies were only detectable by MAIPA. Light chain restriction $(n=9)$ was not associated with HPA-containing antibodies or with any pattern of the HLA antibodies (table 1, fig. 1). Similarly, polyclonal antibodies $(n=8)$ were seen in all categories. These results indicate that pregnancy-associated HLA antibodies can be clonal or polyclonal, irrespective of a diagnosis of assumed FNAITP.

Further work is required to understand why FNAIT develops in some but not all fetomaternal HLA incompatibilities (Elucidation of these mechanisms may also lead to possible prevention of a harmful disorder). Taaning [1] has mentioned some very plausible hypotheses, but most of them have not been further followed experimentally since then. It may also be that in most cases with platelets affected by HLA antibodies, their removal follows a two-component curve with a small component showing a short survival and a larger component with a longer survival, like transfused red cells which are incompatible with HLA antibodies [18]. A slightly reduced life span may be compensated by most fetuses, but not all. Other regulatory proteins that may play a role are FcRn [19], and the generation of HLA antiidiotypes which develop concurrently with the production of HLA antibodies [20].

\section{Conclusion}

The dispute on whether or not HLA antibodies can induce FNAITP will be ongoing, unless a mechanism is shown which protects the offspring from 'harmful' maternal antibodies. This barrier needs to be broken to let HLA antibodies induce FNAITP.

\section{References}

1 Taaning E: HLA antibodies and fetomaternal alloimmune thrombocytopenia: myth or meaningful? Transfus Med Rev 2000;14:275-80.

2 Saito S, Ota M, Komatsu Y, Ota S, Aoki S, Koike K, Tokunaga I, Tsuno T, Tsuruta G, Kubo T, Fukushima H: Serologic analysis of three cases of neonatal alloimmune thrombocytopenia associated with HLA antibodies. Transfusion 2003;43:908-17.

3 Moncharmont P, Dubois V, Obegi C, Vignal M, Merieux Y, Gebuhrer L, Rigal D: HLA antibodies and neonatal alloimmune thrombocytopenia. Acta Haematol 2004;111:215-20.

4 Thude H, Schorner U, Helfricht C, Loth M, Maak B, Barz D: Neonatal alloimmune thrombocytopenia caused by human leucocyte antigen-B27 antibody. Transfus Med 2006;16:143-9.

5 Dreyfus M, Kaplan C, Verdy E, Schlegel N, Durand-Zaleski I, Tchernia G: Frequency of immune thrombocytopenia in newborns: a prospective study. Immune Thrombocytopenia Working Group. Blood 1997;89:4402-6.
6 Williamson LM, Hackett G, Rennie J, Palmer CR, Maciver C, Hadfield R, Hughes D, Jobson S, Ouwehand WH: The natural history of fetomaternal alloimmunization to the platelet-specific antigen HPA-1a (PlA1, Zwa) as determined by antenatal screening. Blood 1998;92:2280-7.

7 Davoren A, Smith G, Lucas G, Rodgers S, O’Donoghue P, Crowley J, Barnes CA, McKiernan J: Neonatal alloimmune thrombocytopenia due to HPA-3a antibodies: a case report. Immunohematology 2002;18:33-6.

8 Schallmoser K, Kutschera J, Macher S, Ulrich S, Eichler P, Panzer S, Lanzer G: Delayed detectability of anti-HPA-3a by the MAIPA assay in a severe neonatal alloimmune thrombocytopenia but successful transfusion of incompatible donor platelets: a case report. Vox Sang 2006;91:181-3.

9 Davoren A, Curtis BR, Aster RH, McFarland JG: Human platelet antigen-specific alloantibodies implicated in 1162 cases of neonatal alloimmune thrombocytopenia. Transfusion 2004;44:1220-5.
10 Panzer S: Report on the Tenth International Platelet Genotyping and Serology Workshop on behalf of the International Society of Blood Transfusion. Vox Sang 2001;80:87-43.

11 Goldman M, Trudel E, Richard L: Report on the Eleventh International Society of Blood Transfusion Platelet Genotyping and Serology Workshop. Vox Sang 2003;83:149-155.

12 Bessos H, Wilson DWL, Metcalfe P, Allen D, Urbaniak SJ: Report on the 12th International Society of Blood Transfusion Platelet Immunology Workshop. Vox Sang 2005;89:105-113.

13 Berry JE, Murphy CM, Smith GA, Ranasinghe E, Finberg R, Walton J, Brown J, Navarrete C, Metcalfe P, Ouwehand WH: Detection of Gov system antibodies by MAIPA reveals an immunogenicity similar to the HPA-5 alloantigens. Br J Haematol 2000;110:735-42.

14 Ertel K, Al-Tawil M, Santoso S, Kroll H: Relevance of the HPA-15 (Gov) polymorphism on CD109 in alloimmune thrombocytopenic syndromes. Transfusion 2005;45:366-73. 
15 Mandelbaum M, Koren D, Eichelberger B, Auerbach L, Panzer S: Frequencies of maternal platelet alloantibodies and autoantibodies in suspected fetal/neonatal alloimmune thrombocytopenia, with emphasis on human platelet antigen-15 alloimmunization. Vox Sang 2005;89:39-43.

16 Koyama N, Ohama Y, Kaneko K, Itakura Y, Nakamura T, Takasaki J, Tanaka T, Eguchi H, Kawase A, Kamiya K, et al: Association of neonatal thrombocytopenia and maternal anti-HLA antibodies. Acta Paediatr Jpn 1991;33:71-6.

17 Panzer S, Mayr WR, Eichelberger B: Light chain phenotypes of HLA antibodies in cases with suspected neonatal alloimmune thrombocytopenia. Vox Sang 2005;89:261-4.

18 Panzer S, Mueller-Eckhardt G, Salama A, Strauss BE, Kiefel V, Mueller-Eckhardt C: The clinical significance of HLA antigens on red cells. Survival studies in HLA-sensitized individuals. Transfusion 1984;24:486-9.

19 de Haas M: IgG-Fc receptors and the clinical relevance of their polymorphisms. Wien Klin Wochenschr 2001;113:825-31.

20 Semple JW, Kim M, Lazarus AH, Freedman J: Gamma-globulins prepared from sera of multiparous women bind anti-HLA antibodies and inhibit an established in vivo human alloimmune response. Blood 2002;100:1055-9. 